\begin{tabular}{|c|c|c|}
\hline $2=-3$ & American Journal of Dermatological Research and Reviews & 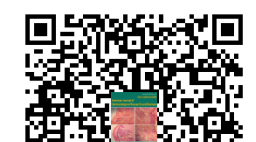 \\
\hline$\frac{1-1}{2-1}=$ & (ISSN:2638-1893) & 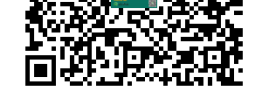 \\
\hline
\end{tabular}

\title{
Dermatological adverse events of immune checkpoint inhibitors
}

\author{
Kevin Yang ${ }^{1}$, Khurram Yusuf ${ }^{2}$ \\ 1Dermatology, UAB School of Medicine, Birmingham, AL 35294 \\ ${ }^{2}$ Kendriya Vidyalaya, Jabalpur, MP 482004, India
}

\begin{abstract}
Treatment with immune checkpoint inhibitors is a landmark in the treatment of melanoma and other cancers. These treatments have been very effective and have increased the survival of cancer patients. The promise of immunotherapy also comes with a variety of adverse events. One of the common sites of immune related adverse events (irAEs) is skin. The cutaneous irAEs present a unique challenge to the success of immunotherapy. It is important to diagnose and understand the mechanism related to these cutaneous irAEs to increase the effectiveness of immune checkpoint inhibitor therapy. In this review, we have characterized the various cutaneous irAEs associated with immune checkpoint inhibitor therapies and their possible mechanisms.
\end{abstract}

How to cite this article:

Kevin Yang, Khurram Yusuf. Dermatological adverse events of immune checkpoint inhibitors.American Journal of Dermatological Research and Reviews, 2020, 3:33.

Keywords: checkpoint inhibitors, immunotherapy, adverse events

*Correspondence to Author:

Kevin Yang

Department of Dermatology, 1670 , University Boulevard, VH 564

Birmingham AL 35294

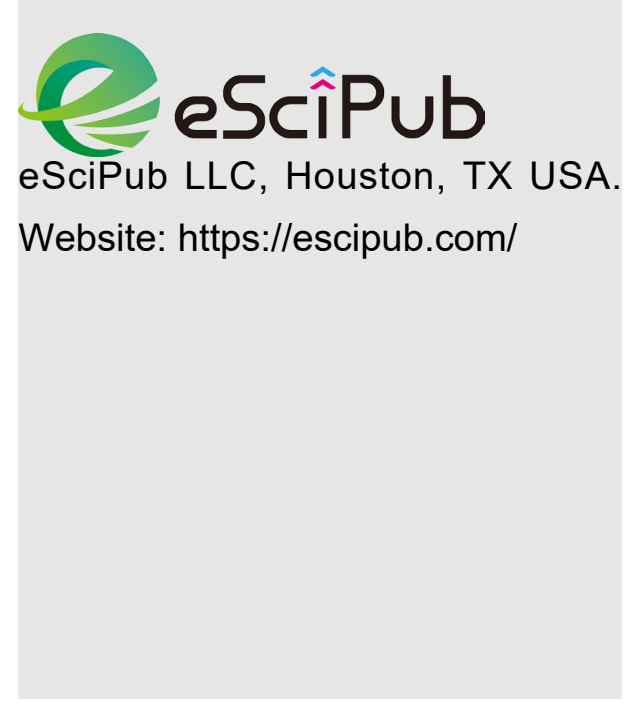




\section{Introduction}

Immune checkpoint inhibitors are increasingly being used in cancer therapy. These drugs work to enhance the immune response against cancer by targeting the intrinsic inactivation signals of the immune system. Specifically, these signals are cytotoxic T-lymphocyte antigen-4 (CTLA-4), programmed death-1 (PD-1), and programmed death-ligand 1 (PD-L1). During activation, T-cells are co-stimulated by antigen presenting cells (APCs) by binding of antigen-major histocompatibility complex (MHC) to the T-cell receptor (TCR) and binding of CD80/86 to the CD28 receptor on T-cells. T-cell activation feeds into a negative feedback loop, whereby CTLA-4 expression increases and can bind to the CD28 receptor with greater affinity than $\mathrm{CD} 80 / 86$. As a result, the T-cell response becomes downregulated ${ }^{[1,2]}$. In the peripheral tissues, binding of PD-1 on T-cells to PD-L1 on normal or tumor cells reduces the activity and proliferation of $\mathrm{T}$ cells $^{[3,4]}$. Therefore, the strategy to block CTLA4 , PD-1, or both has been successfully harnessed in the use of immune checkpoint inhibitors against a variety of cancers.

One of the major downsides of checkpoint blockade is immune-related adverse events (irAEs). These events occur systemically and unpredictably and can lead to serious consequences, including death. Of the many irAEs, the most prevalent reactions occur in the skin with upwards of $30 \%$ of patients manifesting with cutaneous events upon checkpoint inhibitor therapy ${ }^{[5-7]}$. Most cutaneous reactions are relatively mild with rare occurrences of grade 3 or 4 events ${ }^{[8,9]}$. The pathogenesis for these events is not well understood, but most explanations center around over-stimulation of the immune system.

In this review, we will describe the reported cutaneous adverse events and examine the underlying mechanisms for these effects.

\section{Dermatologic Adverse Events}

\section{Maculopapular rash}

A maculopapular rash is a type of rash characterized by a flat erythematous area on the skin covered by small confluent bumps. The most common dermatological and overall irAE is a maculopapular rash (MPR). It is the most commonly seen under blockade of CTLA-4 (4-68\% patients) in comparison to single treatment with PD-1 or PD-L1 inhibitors (20\% patients) [10, 11]. MPR most commonly manifests on the trunk and surface of extremities. It is an early event, which is seen 3-6 weeks after the first treatment and is dependent on the therapeutic dose. Histologically, there is infiltration of CD4+ T-cells and eosinophils in the tissue ${ }^{[12] .}$

\section{Pruritus}

Pruritus is a chronic itchy condition that provokes the desire to scratch. Pruritus is the second most common irAE after MPR and the two are often seen to co-exist. It can occur with or without cutaneous eruption. It is often seen in patients on anti-CTLA4 therapy ${ }^{[13,14]}$. A small number of patients $(\sim 20 \%)$ can also develop pruritis after antiPD-1/PD-L1 therapy ${ }^{[13-16] . ~}$

\section{Lichenoid eruption}

Lichenoid eruption is a condition characterized by damage and infiltration between the epidermis and dermis. Lichenoid eruption is seen with PD-1/PD-L1 therapy, although to a much lower extent $(0.5-6 \%$ patients) compared to MPR $[10,17,18]$. Unlike MPR, its onset is delayed (mean onset is 6-12 weeks). There eruptions appear as multiple, discrete, erythematous, papules and plaques on chest and back region, but they can be rarely seen on limbs, palmoplantar surfaces, and oral mucosa [12]. Histological evaluation reveals a dense superficial dermal band-like lymphocytic infiltrate with degeneration of vacuoles and apoptotic keratinocytes in the basal epidermal layer [12]. These are different from the conventional lichenoid eruptions in that they consist of increased number of CD163-positive cells of macrophage-monocytic lineage ${ }^{[19-21]}$.

\section{Autoimmune Diseases}

\section{Vitiligo}

Vitiligo is an autoimmune disease characterized by depigmented macules or patches that originate due to loss of functional melanocytes in the 


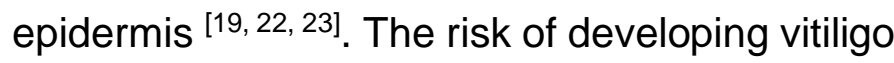
after checkpoint inhibitor therapy is 10 times larger than what is seen in the general population [JR]. Development of vitiligo is a positive indicator of the success of therapy with immune checkpoint inhibitors. Vitiligo develops due to an immune reaction by activated CD8+ cytotoxic $T$ cells against melanocytes after anti-PD- 1 or antiCTLA-4 checkpoint inhibitor therapy [13, 14, 22, 24]. Vitiligo takes several months to develop after initiation of immune checkpoint inhibitor therapy and is not dose-dependent ${ }^{[13,14] .}$. The incidence of vitiligo-like depigmentation in also seen in other non-melanoma related cancers but its incidence is unknown [26-28]. Vitiligo that arises after immune checkpoint inhibitor therapy is seen as multiple flecked macules of depigmentation that evolve into large plaques on the skin ${ }^{\text {[2]. Patients }}$ who develop vitiligo after immune checkpoint inhibitor therapy have a better progression free and disease free survival and it does not resolve even after completion of therapy $[13,14,19]$.

\section{Bullous pemphigoid-like blisters}

Bulbous pemphigoid (BP) is a rare skin autoimmune disease that causes rash and itchy blisters. BP-like blisters arising after PD-1 inhibitor have been identified in a few cases. These cases are similar with the appearance of tense bullae with serous exudate. In addition, histology revealed subepidermal detachment and eosinophilic infiltration ${ }^{[28-33]}$. More rarely, other forms of autoimmune blistering diseases may develop. A case report revealed a patient with paraneoplastic pemphigus-like lesions identified by suprabasal acantholytic dermatoses ${ }^{[34]}$. BP-like blisters are associated with therapy with anti-PD-1/PD-L1 inhibitors for melanoma and other cancers. They have a delayed onset of 14 weeks after therapy $[10,26]$. They have a non-bullous phase of pruritis, followed by localized blisters that are filled with hemorrhagic fluid [35]. A humoral component is involved in the development of BP-like blisters that are characterized by autoantibody production, which can be detected by serological meth- ods like ELISA for monitoring the disease [36]. Indirect immunofluorescence can also be performed for circulating $\lg G$ autoantibodies [12].

\section{Scleroderma}

Scleroderma is a group of rare diseases that involve the hardening and tightening of the skin and connective tissues. Scleroderma is an even rarer event that has been reported following PD1 blockade. These cases involved typical skin tightness of the hands and feet and dermal sclerosis on histology ${ }^{[37,38]}$. The underlying mechanism for this is unknown, it is reported that TGF$\beta$ cascade is activated within the skin after treatment with anti-PD-1, which results in fibrosis ${ }^{[37]}$.

\section{Alopecia areata}

Alopecia areata (AA) is an autoimmune disease that results in unpredictable, patchy hair loss. AA is reported in $1-2 \%$ cancer patients receiving immune checkpoint inhibitor therapy [38, 39]. AA caused by checkpoint inhibitor therapy shares several features with the classical AA. PD-1/PDL1 play an important role in immune privileged sites including the hair follicle [11]. In both conditions, there is an infiltration of CD4+ and CD8+ T-cells sparing the stem cell compartment. There is some difference in lymphocyte infiltration. In immune checkpoint inhibitor-induced AA, lymphocytes are seen around the infundibulum of the hair follicle in addition to the peribulbular region as seen in classical AA [40]. Immune checkpoint therapy can also amplify pathology in patients with pre-existing compromise of hair follicle immune privilege ${ }^{[41]}$.

\section{Systemic Lupus Erythematosus (SLE)}

Systemic Lupus Erythematosus (SLE) is the most common form of lupus, a chronic autoimmune disease that can cause severe fatigue and joint pain. Polymorphisms in CTLA4 and PD1 genes have been shown to cause SLE in humans and animal models ${ }^{[42-44]}$. Manifestation of SLE is rarely seen after immune checkpoint inhibitor therapy in patients ${ }^{[45]}$.

\section{Inflammatory Reactions \\ Psoriasis}


Psoriasis is an immune-mediated disease that causes inflammation in the body characterized by raised scaly patches to appear on the skin, Psoriasis is caused due to an inflammatory reaction to immune checkpoint inhibitors. Most of these cases were precipitated by PD-1 therapy and involved exacerbations in patients with existing psoriasis, but rarely patients have also developed new psoriasis ${ }^{[46-48]}$. Notably, the few cases of psoriasis following CTLA-4 therapy occurred in patients with pre-existing psoriasis ${ }^{[49]}$. The majority of cases are classified as plaque psoriasis, typified by scaly, erythematous plaque lesions on the body [50]. A few cases of guttate and palmoplantar psoriasis have also been reported [51].

\section{Sarcoidosis}

Sarcoidosis is a disease that starts as granuloma and causes inflammation in lungs, skin, or lymph nodes. Sarcoidosis has been reported in several cases after both CTLA-4 and PD-1 blockade with incidence ranging from about 5 $6.7 \%$ of patients ${ }^{[52,53]}$. These events are marked by cutaneous nodules of epithelioid histiocytes and giant cells, as well as hilar and/or mediastinal lymphadenopathy ${ }^{[54-57]}$.

\section{Grover's disease}

Grover disease is a common pruritic condition that presents as erythematous papules or papulovesicles, mostly occurs on the trunk. It may be caused by an inflammatory reaction to altered self-antigens [58]. It is conceivable that immune stimulatory effects of checkpoint inhibitor therapy may result in altered homeostasis of immune system and trigger Grover-like irAE in susceptible patients ${ }^{[59]}$.

\section{Other}

\section{Sweet syndrome}

Sweet syndrome is a very rare skin inflammatory skin condition characterized by a sudden onset of fever and painful rash on the arms, legs, trunk, face or neck. Sweet syndrome has been reported after treatment with anti-CTLA-4 therapy. It occurred early in the course of treatment and was found to be dose dependent [60].

\section{Stevens-Johnson syndrome/toxic epidermal necrolysis (SJS/TEN)}

Stevens-Johnson syndrome (SJS) is a rare serious disorder of the skin and mucous membranes. A more severe form of the condition is called toxic epidermal necrolysis (TEN). Acute SJS/TEN presentations can occur with immune checkpoint inhibitor therapy. They resemble the features of classical SJS/TEN. Most cases are seen in patients with melanoma but they can occur in cases of other cancers as well. Fever and clinical manifestations can occur before appearance of skin lesions, which rapidly manifest on trunk and extremities and are associated with mucosal involvement (ocular, oral or urogenital) [61]. Majority of cases are seen after first or second infusion of anti-PD-1 therapy [62, 63]. The diagnosis of SJS/TEN can be done clinically and histopathologically. Histological evaluation of early lesions show scattered apoptotic keratinocytes in the basal epidermis but more full-thickness epidermal necrosis in more advanced lesions ${ }^{[62,63]}$.

\section{Mechanisms of AEs}

The mechanism of cutaneous irAEs is widely thought to relate to excessive activation of the immune system, which may eventually result in auto-immune conditions. Furthermore, the pathogenesis for CTLA-4 inhibitors and PD-1/PD-L1 inhibitors are different, as illustrated by their contrasting downstream pathways ${ }^{[64]}$. Generally, the adverse events of CTLA-4 blockade are more severe than those of PD-1 blockade[65]. A phase 3 clinical trial showed that nivolumab and ipilimumab combined therapy produced a greater incidence of adverse events than with either treatment alone ${ }^{[6]]}$. This further supports the notion that CTLA-4 and PD-1 act along different pathways and thus carry more potential to cause adverse effects.

\section{Enhanced T-cell response}

When specific tumor T-cell infiltrating populations in reponse to immune checkpoint inhibitors were compared, it was found that anti-PD-1 and anti-CTLA-4 operate through distinct cellular 
mechanisms. PD-1 blockade induces proliferation of CD8 T cells, while CTLA-4 blockade induces proliferation of inducible T-cell co-stimulator (ICOS)+ Th1-like CD4 T cells as well as exhausted-like CD8 T-cells. Furthermore, a correlation was observed between the frequency of CD4+ and CD8+ T-cell populations and tumor growth [64]. In an infection model, it was found that PD-1 blockade can restore the function of exhausted T cells [67]. PD-1 activation has been used to control inflammatory cytokine production in diseases like psoriasis. These cytokines of $T$ helper cells can contribute to ongoing inflammation. T-cell activation by inhibition of PD-1 could possibly exacerbate psoriasis in patients with previous history [51]. Th1 activation also implicated in sarcoidosis. Complete regression of stage IV melanoma after anti-PD-1 therapy was seen in a patient who developed pulmonary and cutaneous sarcoidoisis as a side effect ${ }^{[57]}$. Activation of autoreactive $T$ cells was seen in immune checkpoint inhibitor mediated autoimmune blistering diseases. A possible mechanism could be the epitope spreading from checkpoint inhibitor induced injury causing immune activation and formation of autoreactive T-cells. Another possible mechanism could be the recruitment of immune cells to the skin and dysregulation of cytokines [34].

\section{Enhanced Humoral Immunity}

PD-1 blockade may play role in modulating humoral immunity. Transient hyperthyroidism accompanied by anti-thyroid antibodies was observed after PD-1 therapy for non-small cell lung cancer (NSCLC) and this was associated with improved outcomes. This highlighted the role of antibody-mediated toxicity in T-cell-directed therapy [68]. PD-1 is more broadly expressed than CTLA-4 on B cells. Therefore, it may also enhance humoral immunity indirectly via $T$ cells or

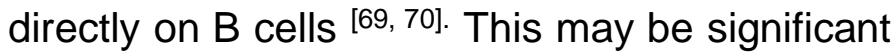
in the case of antibody-mediated autoimmune diseases such as bullous pemphigoid, especially considering that bullous pemphigoid manifested after PD-1 blockade (Naidoo). PD-1 is also ex- pressed on NK cells and inhibits their lytic activity. PD-1 blockade can enhance activity of NK cells is tumors and other tissues ${ }^{[71,72] .}$

\section{Conclusion}

Immunotherapy with immune checkpoint inhibitors have revolutionized the therapeutic approaches for melanoma and other cancers. Most commonly used immune checkpoint inhibitors include PD-1/PD-L1, CTLA-4, and several other emerging candidates. These therapies have been very effective in treating these cancers but are associated with adverse events including the ones associated with skin. These include inflammatory and autoimmune skin conditions caused by hyperactivation of the immune system. More research is needed to investigate the mechanism of action of these cutaneous events so they can be effectively managed for better outcomes.

\section{ACKNOWLEDGEMENT}

This work is supported by grant from Melanoma Research Foundation to Kevin Yang.

\section{REFERENCES}

1. O'Day SJ, Hamid O, Urba WJ. 2007. Targeting cytotoxic T-lymphocyte antigen-4 (CTLA-4): a novel strategy for the treatment of melanoma and other malignancies, Cancer 110(12): 2614-27.

2. Fong L, Small EJ. 2008. Anti-cytotoxic Tlymphocyte antigen-4 antibody: the first in an emerging class of immunomodulatory antibodies for cancer treatment. J Clin Oncol. 26(32): 527583.

3. Zitvogel L, Kroemer G. 2012. Targeting PD-1/PDL1 interactions for cancer immunotherapy, Oncoimmunology 1(8): 1223-5.

4. Freeman $\mathrm{GJ}$, Long $\mathrm{AJ}$, Iwai $\mathrm{Y}$, Bourque $\mathrm{K}$, Chernova T, Nishimura $\mathrm{H}$ et al. 2000. Engagement of the PD-1 immunoinhibitory receptor by a novel B7 family member leads to negative regulation of lymphocyte activation, $J$ Exp Med. 192(7):1027-34.

5. Quirk SK, Shure AK, Agrawal DK. 2015. Immunemediated adverse events of anticytotoxic $T$ lymphocyte-associated antigen 4 antibody therapy in metastatic melanoma, Translational research : J Lab Clin Med. 166(5):412-24.

6. Sibaud V. 2018. Dermatologic Reactions to Immune Checkpoint Inhibitors : Skin Toxicities and Immunotherapy, Am J Clin Dermatol. 19(3):345-361. 
7. Thebeau M, Rubin K, Hofmann M, Grimm J, Weinstein A, Choi JN. 2017. Management of skin adverse events associated with immune checkpoint inhibitors in patients with melanoma: A nursing perspective. J Am Assoc Nurse Pract 29(5):294-303.

8. Robert C, Long GV, Brady B, Dutriaux C, Maio M, Mortier L, Hassel JC et al. 2015. Nivolumab in previously untreated melanoma without BRAF mutation. N Engl J Med. 372(4): 320-30.

9. Weber JS, Kahler KC, Hauschild A (2012). Management of immune-related adverse events and kinetics of response with ipilimumab, Am Soc Clin Oncol. 30(21):2691-7.

10. Curry JL, Tetzlaff MT, Nagarajan P, et al. 2017. Diverse types of dermatologic toxicities from immune checkpoint blockade therapy. J Cutan Pathol. 44(2):158-176.

11. Jaber SH, Cowen EW, Haworth LR, et al. 2006. Skin reactions in a subset of patients with stage IV melanoma treated with anti-cytotoxic T-lymphocyte antigen 4 monoclonal antibody as a single agent. Arch Dermatol. 142(2):166-172.

12. Geisler AN, Phillips GS, Barrios DM, et al. 2020. CME Part II: Immune checkpoint inhibitor-related dermatologic adverse events. J Am Acad Dermatol. S0190-9622(20)30963-4.

13. Sibaud V, Meyer N, Lamant L, Vigarios E, Mazieres J, Delord JP. 2016. Dermatologic complications of anti-PD-1/PD-L1 immune checkpoint antibodies. Curr Opin Oncol. 28(4):254-263.

14. Sibaud V. 2018. Dermatologic reactions to immune checkpoint inhibitors: skin toxicities and immunotherapy. Am J Clin Dermatol. 2018;19(3):345-361.

15. Sanlorenzo M, Vujic I, Daud A, et al. 2015. Pembrolizumab cutaneous adverse events and their association with disease progression. JAMA Dermatol. 151(11):1206-1212.

16. Tetzlaff MT, Nagarajan P, Chon S, et al. 2017. Lichenoid dermatologic toxicity from immune checkpoint blockade therapy: A detailed examination of the clinicopathologic features. Am J Dermatopathol. 39(2):121-129.

17. Phillips GS, Wu J, Hellmann MD, et al. 2019. Treatment outcomes of immune-related cutaneous adverse events. J Clin Oncol. 37(30):27462758.

18. Kaunitz GJ, Loss M, Rizvi H, et al. 2017. Cutaneous eruptions in patients receiving immune checkpoint blockade: Clinicopathologic analysis of the nonlichenoid histologic pattern. Am J Surg Pathol. 41(10):1381-1389.
19. de Golian E, Kwong BY, Swetter SM, Pugliese SB 2016. Cutaneous complications of targeted melanoma therapy. Curr Treat Options Oncol.17 (11):57.

20. Lacouture M, Sibaud V. 2018. Toxic side effects of targeted therapies and immunotherapies affecting the skin, oral mucosa, hair, and nails. Am J Clin Dermatol.19 (Suppl1):31-39.

21. Schaberg KB, Novoa RA, Wakelee HA, et al. 2016. Immunohistochemical analysis of lichenoid reactions in patients treated with anti-PD-L1 and antiPD-1 therapy. J Cutan Pathol. 43:339-346.

22. Hua C, Boussemart L, Mateus C, et al. 2016. Association of vitiligo with tumor response in patients with metastatic melanoma treated with pembrolizumab. JAMA Dermatol. 152(1):45-51.

23. Teulings HE, Limpens J, Jansen SN, et al. 2015. Vitiligo-like depigmentation in patients with stage III-IV melanoma receiving immunotherapy and its association with survival: a systematic review and meta-analysis. J Clin Oncol. 33(7):773-781.

24. Quaglino P, Marenco F, Osella-Abate S, et al. 2010. Vitiligo is an independent favourable prognostic factor in stage III and IV metastatic melanoma patients: results from a single institution hospital-based observational cohort study. Ann Oncol. 21(2):409-414.

25. Phillips GS, Wu J, Hellmann MD, et al. 2019. Treatment outcomes of immune-related cutaneous adverse events. J Clin Oncol. 37 (30):27462758.

26. Larsabal M, Marti A, Jacquemin C, et al. 2017. Vitiligo-like lesions occurring in patients receiving anti-programmed cell death- 1 therapies are clinically and biologically distinct from vitiligo. $\mathrm{J} \mathrm{Am}$ Acad Dermatol. 76(5):863-870.

27. Liu RC, Consuegra G, Chou S, Fernandez Penas P. 2019. Vitiligo-like depigmentation in oncology patients treated with immunotherapies for nonmelanoma metastatic cancers. Clin Exp Dermatol. 44(6):643-646.

28. Lolli C, Medri M, Ricci M, et al. 2018. Vitiligo-like lesions in a patient treated with nivolumab for renal cell carcinoma. Medicine (Baltimore). 97(52):e13810.

29. Anastasopoulou A, Papaxoinis G, Diamantopoulos $P$, Christofidou E, Benopoulou O, Stratigos A, Gogas H. 2018. Bullous pemphigoidlike skin lesions and overt eosinophilia in a patient with melanoma treated with nivolumab: case report and review of the literature, Journal of immunotherapy (Hagerstown, Md. : 1997) 41(3): 164-167.

30. Lomax AJ, Ge L, Anand S, McNeil C, Lowe P. 2016. Bullous pemphigoid-like reaction in a 
patient with metastatic melanoma receiving pembrolizumab and previously treated with ipilimumab, The Austral J Dermatol. 57(4):333335.

31. Parakh S, Nguyen R, Opie JM, Andrews MC. 2017. Late presentation of generalised bullous pemphigoid-like reaction in a patient treated with pembrolizumab for metastatic melanoma. Australas J Dermatol. 58(3):e109-e112.

32. Sowerby L, Dewan AK, Granter S, Gandhi L, LeBoeuf NR (2017). Rituximab treatment of nivolumab-induced bullous pemphigoid. JAMA Dermatol. 153(6) : 603-605.

33. Ridpath AV, Rzepka PV, Shearer SM, Scrape SR, Olencki TE, Kaffenberger BH. 2018. Novel use of combination therapeutic plasma exchange and rituximab in the treatment of nivolumab-induced bullous pemphigoid. Int J Dermatol. 57 (11):13721374.

34. Naidoo J, Schindler K, Querfeld C, Busam K, Cunningham J, Page DB et al. 2016. Autoimmune bullous skin disorders with immune checkpoint inhibitors targeting PD-1 and PD-L1, Cancer Immunol Res. 4(5): 383-9.

35. Chen WS, Tetzlaff MT, Diwan $H$, Jahan-Tigh $R$, Diab A, Nelson $K$ et al. 2018. Suprabasal acantholytic dermatologic toxicities associated checkpoint inhibitor therapy: A spectrum of immune reactions from paraneoplastic pemphigus-like to Grover-like lesions, J Cutaneous Pathol. 45(10):764-773.

36. Lopez AT, Khanna T, Antonov N, Audrey-Bayan C, Geskin L. 2018. A review of bullous pemphigoid associated with PD-1 and PD-L1 inhibitors. Int J Dermatol. 57(6):664-669.

37. Barbosa NS, Wetter DA, Wieland CN, Shenoy NK, Markovic SN, Thanarajasingam U. 2017. Scleroderma Induced by Pembrolizumab: A Case Series. Mayo Clin Proc. 92(7):1158-1163.

38. Tjarks BJ, Kerkvliet AM, Jassim AD, Bleeker JS. 2018. Scleroderma-like skin changes induced by checkpoint inhibitor therapy, J Cutaneous Pathol. 45(8):615-618.

39. Naidoo J, Page DB, Li BT et al. 2015. Toxicities of the anti-PD-1 and anti-PD-L1 immune checkpoint antibodies. Ann Oncol. 26: 2375- 91.

40. Belum VR, Benhuri B, Postow MA et al. 2016. Characterization and management of dermatologic adverse events to agents targeting the PD-1 receptor. Eur J Cancer. 60: 12-25.

41. Zarbo A, Belum VR, Sibaud V, et al. 2017. Immune-related alopecia (areata and universalis) in cancer patients receiving immune checkpoint inhibitors. Br J Dermatol. 176(6):1649-1652.

42. Nishimura $H$, Nose $M$, Hiai $H$, Minato $N$, Honjo $T$. 1999. Development of lupus-like autoimmune diseases by disruption of the PD-1 gene encoding an ITIM motif-carrying immunoreceptor. Immunity. 11: 141-151.

43. Ceeraz S, Nowak EC, Burns CM, Noelle RJ. 2014. Immune checkpoint receptors in regulating immune reactivity in rheumatic disease. Arthritis Res Ther. 16, 1-12.

44. Lu K.-L, Wu M.-Y, Wang C.-H, Wang C.-H, Hung H.-L, Cung W.-H, Chen C.-B. 2019. The role of immune checkpoint receptors in regulating immune reactivity in lupus. Cells 8, 1213 .

45. Arnaud L, Lebrun-Vignes B, Salem J.-E. 2019. Checkpoint inhibitor-associated immune arthritis. Ann Rheum Dis. 78, e68.

46. Ruiz-Banobre J, Abdulkader I, Anido U, Leon L, Lopez-Lopez R, Garcia-Gonzalez J. 2017. Development of de novo psoriasis during nivolumab therapy for metastatic renal cell carcinoma: immunohistochemical analyses and clinical outcome. APMIS 125(3):259-263.

47. Chia PL, John T. 2016. Severe psoriasis flare after anti-programmed death ligand 1 (PD-L1) therapy for metastatic non-small cell lung cancer (NSCLC). J Immunother (Hagerstown, Md. : 1997) 39(5):202-4.

48. Phadke SD, Ghabour R, Swick BL, Swenson A, Milhem M, Zakharia Y. 2016. Pembrolizumab therapy triggering an exacerbation of preexisting autoimmune disease: a report of 2 patient cases. J Investig Med High Impact Case Rep. 4(4):2324709616674316.

49. Johnson DB, Sullivan RJ, Ott PA, Carlino MS, Khushalani NI, Ye F et al. 2016. Ipilimumab therapy in patients with advanced melanoma and preexisting autoimmune disorders. JAMA Oncol. 2(2):234-40.

50. Bonigen J, Raynaud-Donzel C, Hureaux J, Kramkimel N, Blom A, Jeudy G et al. 2017. AntiPD1-induced psoriasis: a study of 21 patients. JEADV 31(5):e254-e257.

51. Voudouri D, Nikolaou V, Laschos K, Charpidou A, Soupos N, Triantafyllopoulou I et al. 2017. AntiPD1/PDL1 induced psoriasis, Curr Prob Cancer 41(6) : 407-412.

52. Bronstein Y, Ng CS, Hwu P, Hwu WJ. 2011. Radiologic manifestations of immune-related adverse events in patients with metastatic melanoma undergoing anti-CTLA-4 antibody therapy. AJR Am J Roentgenol. 197(6):W992w1000. 
53. Tirumani SH, Ramaiya NH, Keraliya A, Bailey ND, Ott PA, Hodi FS. Et al. 2015. Radiographic profiling of immune-related adverse events in advanced melanoma patients treated with ipilimumab, Cancer Immunol Res. 3(10): 1185-92.

54. Birnbaum MR, Ma MW, Fleisig S, Packer S, Amin BD, Jacobson M, McLellan BN. 2017. Nivolumabrelated cutaneous sarcoidosis in a patient with lung adenocarcinoma, JAAD Case Reports 3(3):208-211.

55. Firwana B, Ravilla R, Raval M, Hutchins L, Mahmoud F. 2017. Sarcoidosis-like syndrome and lymphadenopathy due to checkpoint inhibitors, J Oncol Pharm Pract. 23(8):620-624.

56. Danlos FX, Pages C, Baroudjian B, Vercellino L, Battistella M, Mimoun M et al. 2016. Nivolumabinduced sarcoid-like granulomatous reaction in a patient with advanced melanoma. Chest 149(5):e133-6.

57. Yatim N, Mateus C, Charles P. 2018. Sarcoidosis post-anti-PD-1 therapy, mimicking relapse of metastatic melanoma in a patient undergoing complete remission, La Revue de medecine interne 39(2):130-133.

58. Gantz M, Butler D, Goldberg M, Ryu J, McCalmont T, Shinkai K. 2017. Atypical features and systemic associations in extensive cases of Grover disease: a systematic review. J Am Acad Dermatol. 77:952-7.

59. Vastarella M, Fabbrocini G, Sibaud V. 2020. Hyperkeratotic skin adverse events induced by anticancer treatments: a comprehensive review. Drug Saf. 43(5):395-408.

60. Collins LK, Chapman MS, Carter JB, Samie FH. 2017. Cutaneous adverse effects of the immune checkpoint inhibitors. Curr Probl Cancer. 41(2):125-128.

61. Coleman EL, Olamiju B, Leventhal JS. The lifethreatening eruptions of immune checkpoint inhibitor therapy. Clin Dermatol. 2020;38(1):94-104.

62. Hwang A, Iskandar A, Dasanu A. 2019. StevensJohnson Syndrome manifesting late in the course of pembrolizumab therapy. $\mathrm{J}$ Oncol Pharm Pract. 25 (6): 1520-1522.

63. Saw S, Lee HY, Ng QS. 2017. Pembrolizumab-induced Stevens-Johnson syndrome in non-melanoma patients. Eur J Cancer, 81:237-239

64. Wei SC, Levine JH, Cogdill AP, Zhao Y, Anang NAS, Andrews MC et al. 2017. Distinct cellular mechanisms underlie anti-CTLA-4 and anti-PD-1 checkpoint blockade. Cell 170(6):1120-1133.e17.

65. Postow MA, Sidlow R, Hellmann MD. 2018. Immune-related adverse events associated with immune checkpoint blockade. $\mathrm{N}$ Engl $\mathrm{J}$ Med. 378(2) :158-168.

66. Larkin J, Chiarion-Sileni V, Gonzalez R, Grob JJ, Cowey CL, Lao CD. et al. 2015. Combined nivolumab and ipilimumab or monotherapy in untreated melanoma. N Engl J Med. 373(1) : 2334.

67. Barber DL, Wherry EJ, Masopust D et al. 2006. Restoring function in exhausted CD8 T cells during chronic viral infection. Nature. 439(7077):682687.

68. Osorio JC, Ni A, Chaft JE, et al. 2017. Antibodymediated thyroid dysfunction during T-cell checkpoint blockade in patients with non-small-cell lung cancer. Ann Oncol. 28(3):583-589.

69. Terme $M$. et al. 2011. IL-18 induces PD-1-dependent immunosuppression in cancer. Cancer Res. 71:5393-5399.

70. Fanoni $D$, et al. 2011. New monoclonal antibodies against B-cell antigens: possible new strategies for diagnosis of primary cutaneous B-cell lymphomas. Immunol Lett. 134:157-160.

71. Velu V, et al. 2009. Enhancing SIV-specific immunity in vivo by PD-1 blockade. Nature. 458:206-210.

72. Pardoll DM. 2012. The blockade of immune checkpoints in cancer immunotherapy. Nat Rev Cancer. 12(4):252-264. 